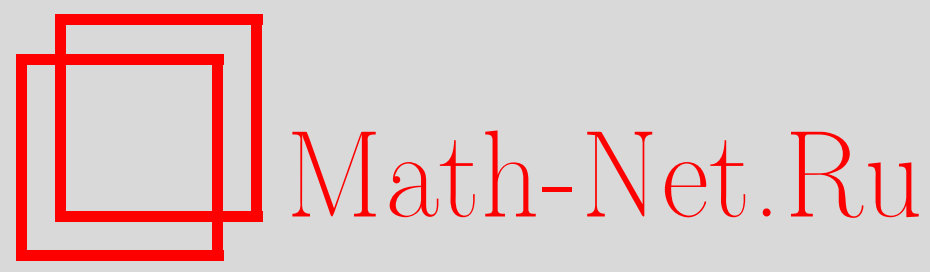

А. В. Филиновский, Убывание решений волнового уравнения и спектральные свойства оператора Лапласа в расширяющихся областях, Матем. заметки, 1998, том 63, выпуск $1,154-156$

DOI: https://doi.org/10.4213/mzm1262

Использование Общероссийского математического портала Math-Net.Ru подразумевает, что вы прочитали и согласны с пользовательским соглашением http://www . mathnet.ru/rus/agreement

Параметры загрузки:

IP : 54.224 .60 .19

26 апреля 2023 г., 13:32:23

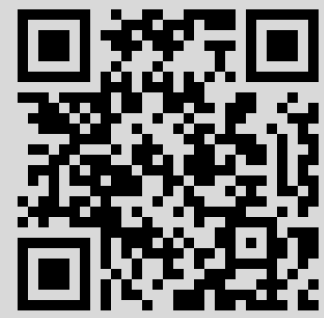




\section{УБЫВАНИЕ РЕШЕНИЙ ВОЛНОВОГО УРАВНЕНИЯ И СПЕКТРАЛЬНЫЕ СВОЙСТВА ОПЕРАТОРА ЛАПЛАСА В РАСШИРЯЮЩИХСЯ ОБЛАСТЯХ}

\section{А. В. Филиновский}

Пусть $\Omega \subset \mathbb{R}^{n}, n \geqslant 2,-$ неограниченная область с границей $\Gamma \in C^{2}$. Рассмотрим задачу

$$
\begin{gathered}
u_{t t}-\Delta u=0, \quad t>0, \quad x \in \Omega, \\
u(0, x)=f(x), \quad u_{t}(0, x)=g(x),\left.\quad u\right|_{\Gamma}=0,
\end{gathered}
$$

с вещественнозначными начальными функциями $f(x) \in \stackrel{\circ}{W}_{2}^{1}(\Omega)$ и $g(x) \in L_{2}(\Omega)$. Хорошо известно, что задача (1) имеет единственное решение из энергетического класса (см., например, [1]), и для него справедлив закон сохранения энергии

$$
E_{\Omega}(t)=\int_{\Omega}\left(u_{t}^{2}+|\nabla u|^{2}\right) d x=E_{\Omega}(0), \quad t>0 .
$$

Говорят, что энергия $E_{\Omega}(t)$ рассеивается на бесконечность, если для любой ограниченной области $\Omega^{\prime} \subset \Omega$ выполнено соотношение

$$
\lim _{t \rightarrow \infty} E_{\Omega^{\prime}}(t)=0
$$

где

$$
E_{\Omega^{\prime}}(t)=\int_{\Omega^{\prime}}\left(u_{t}^{2}+|\nabla u|^{2}\right) d x
$$

Соотношение (2) установлено для произвольнй ограниченной $\Gamma$ [2]. Если $\Gamma$ неограничена, то рассеяние энергии на бесконечность доказано при дополнительных условиях на $\Gamma$. А именно, в работах [3], [4] соотношение (2) установлено в предположении, что поверхность $Г$ звездна относительно начала координат, т.е.

$$
(\nu, x) \leqslant 0, \quad x \in \Gamma,
$$

где $\nu=\left(\nu_{1}, \ldots, \nu_{n}\right)$ - единичный вектор внешней по отношению к $\Omega$ нормали к $\Gamma$. В работе [5] соотношение (2) получено для кривой $\Gamma$, звездной вне некоторого круга $(n=2)$ и степенным образом приближающейся к сторонам угла.

Нас будет интересовать связь между рассеянием энергии решения задачи (1) на бесконечность и спектральными свойствами соответствующей эллиптической краевой задачи.

Пусть $L_{0}$ - минимальный оператор, порожденный в $L_{2}(\Omega)$ дифференциальным выражением $-\Delta$, $L$ - самосопряженное расширение $L_{0}$, соответствуюшее однородному граничному условию Дирихле. Тогда $\sigma(L)=\sigma_{\mathrm{p}}(L) \cup \sigma_{\mathrm{c}}(L) \subset[0,+\infty)$, где $\sigma_{\mathrm{p}}(L)$ - точечный, а $\sigma_{\mathrm{c}}(L)$ - непрерывньй спектр оператора $L$. Оператор назьвается спектрально непрерывным, если его точечный спектр пуст, и спектрально разрывным в противоположном случае.

УТВЕРЖДЕНИЕ 1. Если оператор L спектрально разрывен, то существуют начальнье функиии $f(x) \in \check{C}^{\infty}(\Omega), g(x) \in \mathcal{C}^{\infty}(\Omega)$ и ограниченная область $\Omega^{\prime} \subset \Omega$ такие, ито

$$
\varliminf_{t \rightarrow \infty} E_{\Omega^{\prime}}(t)>0 \text {. }
$$

Таким образом, спектральная непрерывность оператора $L$ является необходимьм условием рассеяния на бесконечность энергии решения задачи (1) с произвольными финитными началньми функциями.

При ограниченной Г оператор $L$ спектрально непрерывен [6]. Сформулируем известные достаточные условия спектральной непрерьвности оператора $L$ в случае неограниченной $\Gamma$. В работе [6] рассмотрен класс областей $\Omega$, граница которых удовлетворяет условию

$$
\left(\nu, e_{n}\right) \leqslant 0, \quad x \in \Gamma,
$$


где $e_{n}$ - координатный вектор оси $O x_{n}$, и таких, что $\operatorname{diam}\left(\Omega \cap\left\{x_{n}<R\right\}\right)<\infty$ при всех $R>0$. Для областей из этого класса, каждая из которых содержит некоторый полуцилиндр, в [6] доказана спектральная непрерьвность оператора $L$. Из результатов работы [7] следует, что $L$ спектрально непрерьвен, если поверхность Г звездна относительно начала координат. Нарушение условия (3) внутри некоторого круга $(n=2)$ не изменяет спектралной непрерывности $L$ [5], а невыполнение (4) на сколь угодно малом участке $\Gamma$ может привести к появлению собственного значения у $L[6]$. В работе [8] был определен класс областей $\Omega$, граница которых вне некоторого шара удовлетворяет условию

$$
\alpha\left(\nu_{1} x_{1}+\cdots+\nu_{n-1} x_{n-1}\right)+\nu_{n} x_{n} \leqslant 0, \quad x \in \Gamma, \quad 0<\alpha<1,
$$

таких, что $\operatorname{diam}\left(\Omega \cap\left\{x_{n}=R\right\}\right) \leqslant C R^{\alpha}, C>0, R>0$, и доказана спектральная непрерьвность оператора $L$. Области, удовлетворяющие (5), содержат некоторый параболоид и по минимальной скорости расширения на бесконечности занимают промежуточное положение между областями со звездньми границами и областями, удовлетворяющими условию (4).

В настоящей работе изучаются области с границами, звездными относительно некоторых классов векторных полей.

Пусть

$$
0<\alpha_{1} \leqslant \alpha_{2} \leqslant \ldots \leqslant \alpha_{n}=1, \quad \varphi(x)=\sum_{j=1}^{n} \alpha_{j} x_{j}^{2} .
$$

ОПРЕДЕЛЕНИЕ 1 . Область $\Omega$ называется областью класса $\widetilde{D}_{\varphi}$, если

$$
(\nu, \nabla \varphi(x)) \leqslant 0, \quad x \in \Gamma \cap\left\{r \geqslant R_{0}\right\}, \quad r=|x|, \quad R_{0}>0 .
$$

Геометрически условие (6) означает звездность поверхности Г относительно векторного поля $\nabla \varphi(x)$. В частности, при $\alpha_{1}=1$ из (6) следует звездность поверхности относительно начала координат. Классы областей $\widetilde{D}_{\varphi}$ для $\alpha_{1}=\cdots=\alpha_{m}=\alpha, \alpha_{m+1}=\cdots=\alpha_{n}=1,1 \leqslant m \leqslant n-1$, были определены в работе [9].

Теорема 1. Ecлu $n \geqslant 2$ u $\Omega \in \widetilde{D}_{\varphi}$, mo оператор $L$ спектрально непрерывен и его спектр заполняет всю полохительную полуось.

Для спектрально непрерывного оператора $L$ из результатов монографиии [2] следует, что при всех $f(x) \in W_{2}^{1}(\Omega), g(x) \in L_{2}(\Omega)$ и произвольной ограниченной области $\Omega^{\prime} \subset \Omega$ выполнено соотношение

$$
\varliminf_{t \rightarrow \infty} E_{\Omega^{\prime}}(t)=0 .
$$

В настоящей работе устанавливается более сильный результат.

УТВеРЖДЕнИЕ 2. Если оператор L спектрально непрерывен, то для любых начальных функиий $f(x) \in \stackrel{\circ}{W}_{2}^{1}(\Omega), g(x) \in L_{2}(\Omega)$ и произвольной ограниченной области $\Omega^{\prime} \subset \Omega$ выполнено соотношение

$$
\lim _{t \rightarrow \infty} \frac{1}{t} \int_{0}^{t} E_{\Omega^{\prime}}(\tau) d \tau=0 .
$$

Из теоремы 1 и утверждения 2 следует теорема 2.

TЕорема 2. Если $n \geqslant 2$ и $\Omega \in \widetilde{D}_{\varphi}$, то для любъх начальных функиий $f(x) \in \stackrel{\circ}{W}_{2}^{1}(\Omega)$, $g(x) \in L_{2}(\Omega)$ и произвольной ограниченной области $\Omega^{\prime} \subset \Omega$ выполнено соотношение (7).

Установим теперь достаточное условие вьполнения более сильного, чем (7), соотношения (2). Для этого сузим класс спектрально непрерьвных операторов $L$. Пусть $\{E(\lambda)\}$ - спектральное семейство проекторов, соответствуюших оператору $L$. Подпространство $H_{\text {ac }} \subset L_{2}(\Omega)$ назьвается подпространством абсолютной непрерывности оператора $L$, если функция $(E(\lambda) h, h)_{L_{2}(\Omega)}$ абсолютно непрерьвна по $\lambda$ для всех $h \in H_{\text {ac }}$ (см., например, [10]). В случае $H_{\text {ac }}=L_{2}(\Omega)$ оператор $L$ называется спектрально абсолютно непрерывным, а его спектр $\sigma_{\mathrm{c}}(L)=\sigma_{\mathrm{ac}}(L)-$ абсолютно непрерывным. 
УТВЕРЖДЕнИЕ 3. Если оператор L спектрально абсолютно непрерывен, то для любых начальных функций $f(x) \in \stackrel{\circ}{W}_{2}^{1}(\Omega), g(x) \in L_{2}(\Omega)$ и произвольной ограниченной области $\Omega^{\prime} \subset \Omega$ выполнено соотношение (2).

Выделим класс областей $\Omega \in \widetilde{D}_{\varphi}$, для которых оператор $L$ спектрально абсолютно непрерывен. Пусть $0<\alpha_{1} \leqslant \ldots \leqslant \alpha_{n-2}<\alpha_{n-1}<\alpha_{n}=1$. Будем называть

$$
d_{m}(R)=\sup _{\Omega \cap\left\{\left|x_{n}\right|=R\right\}}\left(\sum_{j=1}^{m} x_{j}^{2}\right)^{1 / 2}, \quad m=1, \ldots, n-1, \quad R>0,
$$

диаметрами сечения $\Omega \cap\left\{\left|x_{n}\right|=R\right\}$.

Функции $d_{m}(R), R>0$, характеризуют скорость расширения области $\Omega$ по "незвездным" переменным $x_{1}, \ldots, x_{m}$, когда "звездная" переменная $x_{n}$ стремится к бесконечности. Например, для области

$$
\Omega=\left\{x: x_{3}>\left(x_{1}^{2 / \alpha_{1}}+x_{2}^{2 / \alpha_{2}}\right)^{1 / 2}\right\} \subset \mathbb{R}^{3}, \quad 0<\alpha_{1}<\alpha_{2}<1,
$$

имеем $d_{1}(R)=R^{\alpha_{1}}, R^{\alpha_{2}} \leqslant d_{2}(R) \leqslant \sqrt{2} R^{\alpha_{2}}, R>1$.

ОПРЕДЕЛЕНИЕ 2 . Область $\Omega \in \widetilde{D}_{\varphi}$ назьвается областью класса $\widetilde{G}_{\varphi}$, если существует постоянная $C>0$ такая, что

$$
d_{n-2}(R) \leqslant C R^{\alpha_{n-2}}, \quad d_{n-1}(R) \leqslant C R^{\alpha_{n-1}}, \quad x \in \Omega \cap\left\{r \geqslant R_{0}\right\}, \quad R_{0}>0 .
$$

TEOPEMA 3. Ecлu $n \geqslant 2, \Omega \in \widetilde{G}_{\varphi}$

$$
\begin{gathered}
\alpha_{1}>\frac{1}{2} \\
\alpha_{n-1}<\frac{1+3 \alpha_{1}}{2\left(1+\alpha_{1}\right)},
\end{gathered}
$$

то оператор L спектрально абсолютно непрерывен.

Условие (8) означает, что область $\Omega$ расширяется быстрее квадратичного параболоида. Из условия (9) следует, что скорости расшшрения области $\Omega$ по различным "незвездньм" переменным не должны сильно отличаться друг от друга.

Из утверждения 3 и теоремы 3 следует рассеяние на бесконечность энергии решения задачи (1) для областей из классов $\widetilde{G}_{\varphi}$.

ТЕОРема 4. Если выполнены условия теоремы 3 , то для любых начальных функций $f(x) \in \stackrel{\circ}{W}_{2}^{1}(\Omega), g(x) \in L_{2}(\Omega)$ и произвольной ограниченной области $\Omega^{\prime} \subset \Omega$ выполнено соотношение (2).

\section{СПИСОК ЦИТИРОВАННОЙ ЛИТЕРАТУРЫ}

1. Ладыженская О.А. Краевые задачи математической физики. М.: Наука, 1973. 2. Лакс П., Филлипс Р. Теория рассеяния. М.: Мир, 1971. 3. Zachmanoglou E. C. // Arch. Rational Mech. Anal. 1963. V. 14. № 3. Р. 312-325. 4. Муравей Л. А. // Тр. МИАН. 1988. Т. 185. C. 171-180. 5. Рамм А. Г. // Матем. сб. 1965. Т. 66. № 3. C. 321-343. 6. Rellich F. // Jahresber. Deutsch. Math.-Verein. 1943. V. 53. №1. P. 57-65. 7. Odeh F. M. // J. Math. Mech. 1963. V. 12. №6. Р. 857-867. 8. Tayoshi Т. // Publ. Res. Inst. Math. Sci. 1972. V. 8. №2. Р. 375-391. 9. Филиновский А. В. // Матем. сб. 1996. Т. 187. №6. С. 131-160. 10. Като Т. Теория возмущений линейных операторов. М.: Мир, 1972. 